\title{
Prevalence of diabetes and impaired fasting glucose in Uygur children of Xinjiang, China
}

\author{
J. Zhang ${ }^{1}$, Y.T. Ma ${ }^{1}$, X. Xie ${ }^{1}$, Y.N. Yang ${ }^{1}$, F. Liu' ${ }^{2}$, X.M. Li ${ }^{1}$, Z.Y. Fu ${ }^{1}$, \\ X. Ma ${ }^{1}$, B.D. Chen ${ }^{2}$, Y.Y. Zheng ${ }^{1}$ Z.X. Yu ${ }^{1}$ and Y. Chen ${ }^{1}$ \\ ${ }^{1}$ Department of Cardiology, \\ First Affiliated Hospital of Xinjiang Medical University, Urumqi, China \\ ${ }^{2}$ Xinjiang Key Laboratory of Cardiovascular Disease Research, \\ Urumqi, China \\ Corresponding author: Y.T. MA \\ E-mail:myt-xj@163.com
}

Genet. Mol. Res. 12 (4): 5007-5012 (2013)

Received March 28, 2013

Accepted September 10, 2013

Published October 24, 2013

DOI http://dx.doi.org/10.4238/2013.October.24.13

\begin{abstract}
This study was designed to estimate the prevalence of diabetes and impaired fasting glucose (IFG) in Xinjiang children in western China. Data were obtained from the Chun-Miao Project, a community-based, cross-sectional study designed to investigate the prevalence and risk factors of diabetes in children of the Chinese Uygur population in Xinjiang from February 2010 to May 2012. A total of 3644 children completed the survey and measurements of fasting glucose. Diabetes and IFG were defined using American Diabetes Association 2009 criteria. Overall, $0.7 \%$ of the 3644 Uygur children had IFG and $0.1 \%$ had diabetes. In the newborn to 8-year-old group, the prevalence of diabetes and IFG was 0.6 and $1.1 \%$, respectively. In the 9-13-year-old group, the prevalence of diabetes and IFG was 0.1 and $0.7 \%$, respectively. There was no evidence of IFG or diabetes in the 14-17-year-old group. Logistic regression analysis suggested that overweight and obesity were independent risk factors of diabetes in Uygur children of Xinjiang. The prevalence of diabetes and IFG in Uygur children was lower than that reported previously in children of other ethnicities in China.
\end{abstract}

Key words: Children; Prevalence of diabetes; Impaired fasting glucose; Diabetes 


\section{INTRODUCTION}

Diabetes and impaired fasting glucose (IFG) are recognized as risk factors for cardiovascular disease (Ricks et al., 2012). The World Health Organization (WHO) forecasted that the global population will increase by $37 \%$ between 2000 and 2030, and that the number of diabetes patients will increase by $114 \%$ by 2030 (Wild et al., 2004). The International Diabetes Federation (2006) estimated that the number of diabetes patients will likely increase to up to 380 million by 2025. Furthermore, the number of children with type 2 diabetes has increased two to three times in the last 30 years (Berhan et al., 2011). In China, the prevalence of diabetes and pre-diabetes is as high as $9.7 \%$ in adults $\geq 20$ years of age (Yang et al., 2010). In a previous study, we reported that the prevalence of adult diabetes in the Xinjiang Uygur population of western China was 6.23\% (Yang et al., 2012). Tao et al. (2008) reported that the prevalence of diabetes was $8.16 \%$ in the Uygur population and was $1.47 \%$ in the Kazak population in 2008 , whereas Awuti et al. (2012) reported a diabetes prevalence of $9.0 \%$ in the Uygur population in 2010. Fu et al. (2007) found that the prevalence of diabetes in Chinese children and adolescents was $0.19 \%$, and Cao et al. (2007) found total age-adjusted prevalence rates of diabetes and IFG of 0.57 and $1.35 \%$ in Beijing children. Children with IFG or diabetes are at increased risk of developing cardiovascular disease in later life (Berenson et al., 1998; Freedman et al., 2001). However, the prevalence of diabetes and IFG in Uygur children has not yet been reported to date.

In the preset study, we aimed to investigate the prevalence of diabetes and IFG in Uygur children of Xinjiang, in western China.

\section{MATERIAL AND METHODS}

\section{Ethics statement}

The present study was conducted in accordance with the Declaration of Helsinki guidelines, and informed consent was obtained from each individual and guardian according to a protocol approved by the Ethics Committee of the First Affiliated Hospital of Xinjiang Medical University.

\section{Subjects}

From February 2010 to May 2012, we used a random sampling method to select a representative sample of the general population of Chinese Uygur children aged $<18$ years in Xinjiang, China. Three cities (Hetian, Aletai and Kashi) were chosen, and based on the government record of registered residence, one child was randomly selected from each household. In this way, 3644 participants were randomly selected from 31 villages of these three cities and were invited to participate.

Data collection was conducted in examination centers at local hospitals in the participants' residential areas. During clinic or home visits, trained research staff administered a standard questionnaire. Information on demographic characteristics, including age, gender, education, address, and ethnicity, was collected. Height and body weight were measured as described previously (Xie et al., 2010b). Body mass index was calculated by dividing weight $(\mathrm{kg})$ by height $\left(\mathrm{m}^{2}\right)$. Obese and overweight were defined according to criteria suggested by Wu et al. (2010). 
We measured the serum concentration of total cholesterol, triglycerides, blood urea nitrogen, creatinine, low-density lipoprotein, high-density lipoprotein, and fasting glucose by using a chemical analysis equipment (Dimension AR/AVL Clinical Chemistry System, Newark, NJ, USA) at the Clinical Laboratory Department of the First Affiliated Hospital of Xinjiang Medical University as described previously (Xie et al., 2010a, 2011). We defined diabetes using the American Diabetes Association (2009) criteria of fasting plasma glucose (FPG) $\geq 126 \mathrm{mg} / \mathrm{dL}$, or based on self-reported current diabetes treatments in the survey. IFG was defined as $100 \leq \mathrm{FPG} \leq 125 \mathrm{mg} / \mathrm{dL}$.

\section{Data management and statistical analysis}

All questionnaire data were double-entered and cross-validated using EpiData version 3.1 (EpiData Association, Odense, Denmark). Statistical analyses were performed in SPSS version 17.0 (SPSS Institute, Chicago, IL, USA). Continuous variables were summarized with mean and median, and percentages were calculated for categorical variables. The $\chi^{2}$ test was used to explore associations of categorical data. The Student $t$-test was used to compare means of numerical data. Logistic regression analysis was used to calculate the odds ratio and its $95 \%$ confidence interval. Two-tailed tests were performed with the significance level set to 0.05 .

\section{RESULTS}

\section{Characteristics of study participants}

The study cohort consisted of 3644 subjects (1754 males, 1890 females). The age distribution of the study population is shown in Table 1, and the characteristics of participants are shown in Table 2.

Table 1. Gender and age distribution of the participants.
\begin{tabular}{lcc}
\hline Age-group (years) & Male (N, \%) & Female (N, \%) \\
\hline $0-8$ & $179(10.2 \%)$ & $238(12.6 \%)$ \\
$9-13$ & $1518(86.5 \%)$ & $1623(85.9 \%)$ \\
$14-17$ & $57(3.3 \%)$ & $29(1.5 \%)$ \\
Total & 1754 & 1890 \\
\hline
\end{tabular}

\begin{tabular}{|c|c|}
\hline Characteristics & Means $\pm \mathrm{SD}$ \\
\hline Age (years) & $10.64 \pm 1.73$ \\
\hline Waist circumference $(\mathrm{cm})$ & $60.99 \pm 7.48$ \\
\hline Abdominal circumference $(\mathrm{cm})$ & $63.96 \pm 8.30$ \\
\hline Hip circumference $(\mathrm{cm})$ & $71.42 \pm 7.74$ \\
\hline BMI $\left(\mathrm{kg} / \mathrm{m}^{2}\right)$ & $16.58 \pm 2.17$ \\
\hline $\mathrm{SBP}(\mathrm{mmHg})$ & $98.28 \pm 13.26$ \\
\hline DBP $(\mathrm{mmHg})$ & $60.36 \pm 12.72$ \\
\hline $\mathrm{TC}(\mathrm{mM})$ & $2.84 \pm 0.68$ \\
\hline $\mathrm{TG}(\mathrm{mM})$ & $0.81 \pm 0.40$ \\
\hline HDL-C (mM) & $1.04 \pm 0.39$ \\
\hline LDL-C (mM) & $1.50 \pm 0.51$ \\
\hline
\end{tabular}




\section{Prevalence of diabetes and IFG}

Overall, $0.7 \%$ of the 3644 Uygur children had IFG and $0.1 \%$ had diabetes. In the newborn to 8 -year-old group, the prevalence of diabetes and IFG was 0.6 and $1.1 \%$, respectively. In the 9-13-year-old group, the prevalence of diabetes and IFG was 0.1 and $0.7 \%$, respectively. There was no evidence of IFG or diabetes in the 14-17-year-old group (Table 3).

\begin{tabular}{|c|c|c|c|c|c|c|c|c|c|}
\hline \multirow[t]{2}{*}{ Age-group (years) } & \multicolumn{3}{|c|}{ Female (N, \%) } & \multicolumn{3}{|c|}{ Male $(\mathrm{N}, \%)$} & \multicolumn{3}{|c|}{ Total } \\
\hline & $\mathrm{N}$ & Diabetes & $\overline{\mathrm{IFG}(\%)}$ & $\mathrm{N}$ & DM (\%) & IFG (\%) & $\mathrm{N}$ & DM (\%) & IFG (\%) \\
\hline $0-8$ & 238 & $0(0 \%)$ & $4(1.7 \%)$ & 179 & $1(6 \%)$ & $2(1.1 \%)$ & 417 & $1(0.2 \%)$ & $6(1.4 \%)$ \\
\hline $9-13$ & 1623 & $1(0.1 \%)$ & $8(0.5 \%)$ & 1518 & $1(0.1 \%)$ & $10(0.7 \%)$ & 3141 & $2(0.1 \%)$ & $18(0.6 \%)$ \\
\hline $14-17$ & 29 & $0(0 \%)$ & $0(0 \%)$ & 57 & $0(0 \%)$ & $0(0 \%)$ & 86 & $0(0 \%)$ & $0(0 \%)$ \\
\hline Total & 1890 & $1(0.1 \%)$ & $12(0.6 \%)$ & 1754 & $2(0.1 \%)$ & $12(0.7 \%)$ & 3644 & $3(0.1 \%)$ & $24(0.7 \%)$ \\
\hline
\end{tabular}

\section{Risk factors of diabetes in the Uygur population}

Logistic regression analysis revealed two independent risk factors for diabetes in Uygur children (Table 4).

Table 4. Logistic regression analysis.
\begin{tabular}{lcccccr} 
\\
Variables & $\beta$ & SE & Wald & P & OR & 95\%CI \\
\hline Gender & 0.084 & 0.369 & 0.052 & 0.82 & 1.088 & $0.528-2.242$ \\
SBP & 0.009 & 0.02 & 0.183 & 0.669 & 1.009 & $0.969-1.050$ \\
BMI & & & 6.955 & 0.031 & & \\
Overweight & 1.045 & 0.523 & 4 & 0.045 & 2.844 & $1.021-7.919$ \\
Obesity & 1.377 & 0.667 & 4.263 & 0.039 & 3.963 & $1.072-4.647$ \\
TG & 0.045 & 0.413 & 0.012 & 0.913 & 1.046 & $0.466-2.315$ \\
TC & 0.488 & 0.269 & 3.274 & 0.07 & 1.628 & $0.960-2.761$ \\
\hline
\end{tabular}

\section{DISCUSSION}

In the present study, we found that the prevalence of IFG and diabetes was 0.7 and $0.1 \%$, respectively, in Uygur children of Xinjiang, in the west of China. To the best of our knowledge, this is the first study to investigate the prevalence of diabetes in Uygur children.

As described previously, the prevalence of diabetes in Xinjiang adults is very high, and the rate of awareness, treatment, and control is very low, particularly in the Uygur and Kazak populations (Yang et al., 2012). The Xinjiang Province of China is part of the ancient Silk Road and borders eight countries including Russia, Kazakhstan, Kirghizastan, Tajikistan, Pakistan, Mongolia, India, and Afghanistan. There are more than 13 different ethnic groups living in this area. Among them, the Uygur people account for $46 \%$, the Han account for $40 \%$, and Kazaks account for 7\%. Xinjiang is a multi-ethnic region, and Uygur adults are a potentially high-risk diabetic population (Yang et al., 2012). Therefore, much attention must be paid to the children of this population to take preventive measures.

In the present study, we provide the first report of the prevalence of diabetes and IFG in Xinjiang Uygur children. We found prevalence of $0.7 \%$ diabetes and $1.1 \%$ IFG, which are 
lower than rates previously reported for Chinese Han children (Fu et al., 2007; Cao et al., 2007). Logistic regression analysis revealed that overweight and obese status were independent risk factor for diabetes in Xinjiang Uygur children. In addition, we did not find any cases of diabetes or IFG in children in the 14-17-year-old group; however, this result may be due to the very small sample size of this group.

Interestingly, although the prevalence of diabetes in Uygur adults is generally higher than that of other ethnic populations, we found that the prevalence of diabetes and IFG in Uygur children was significantly lower than that of other ethnic populations. This fact suggested that acquired lifestyle and dietary habits are likely the most important predisposing factors of diabetes in the Uygur population. In our analysis, overweight and obesity were independent risk factors of diabetes, suggesting that obesity-related education and popularization of obesity prevention programs should be actively carried out, especially for children.

Our study has several limitations. On the one hand, the present study analyzed only crosssectional data, which did not permit further study of risk factors for incident diabetes. On the other hand, there were very few participants younger than 8 years old and older than 13 years, which might have resulted in underestimation of the prevalence of diabetes in these two age groups.

\section{ACKNOWLEDGMENTS}

Research supported by the Xinjiang Autonomous Region Science and Technology Projects (grant \#201233138).

\section{Conflicts of interest}

The authors declare not conflict of interest.

\section{REFERENCES}

American Diabetes Association (2010). Diagnosis and classification of diabetes mellitus. Diabetes Care 33 (Suppl 1): S62-S69.

Awuti G, Younusi K, Li L, Upur H, et al. (2012). Epidemiological survey on the prevalence of periodontitis and diabetes mellitus in Uyghur adults from rural Hotan area in Xinjiang. Exp. Diabetes Res. 2012: 758921.

Berenson GS, Srinivasan SR, Bao W, Newman WP III, et al. (1998). Association between multiple cardiovascular risk factors and atherosclerosis in children and young adults. The Bogalusa Heart Study. N. Engl. J. Med. 338: 1650-1656.

Berhan Y, Waernbaum I, Lind T, Mollsten A, et al. (2011). Thirty years of prospective nationwide incidence of childhood type 1 diabetes: the accelerating increase by time tends to level off in Sweden. Diabetes 60: 577-581.

Cao BY, Mi J, Gong CX, Cheng H, et al. (2007). The prevalence of diabetes in children and adolescents of Beijing. Zhonghua Liu Xing. Bing. Xue Za Zhi. 28: 631-634.

Freedman DS, Khan LK, Dietz WH, Srinivasan SR, et al. (2001). Relationship of childhood obesity to coronary heart disease risk factors in adulthood: the Bogalusa Heart Study. Pediatrics 108: 712-718.

Fu P, Man Q, Zhang J and Wang C (2007). Epidemiological study on diabetes mellitus in Chinese children and adolescents at the age of 5 to 17 years. Wei Sheng Yan. Jiu. 36: 722-724.

International Diabetes Federation (2006). Diabetes Atlas. 3rd edn. International Diabetes Federation, Brussels.

Ricks J, Molnar MZ, Kovesdy CP, Shah A, et al. (2012). Glycemic control and cardiovascular mortality in hemodialysis patients with diabetes: a 6-year cohort study. Diabetes 61: 708-715.

Tao Y, Mao X, Xie Z, Ran X, et al. (2008). The prevalence of type 2 diabetes and hypertension in Uygur and Kazak populations. Cardiovasc. Toxicol. 8: 155-159.

Wild S, Roglic G, Green A, Sicree R, et al. (2004). Global prevalence of diabetes: estimates for the year 2000 and projections for 2030. Diabetes Care 27: 1047-1053. 
Wu L, Xi B, Zhang M, Shen Y, et al. (2010). Associations of six single nucleotide polymorphisms in obesity-related genes with BMI and risk of obesity in Chinese children. Diabetes 59: 3085-3089.

Xie X, Ma YT, Yang YN, Fu ZY, et al. (2010a). Polymorphisms in the SAA1/2 gene are associated with carotid intima media thickness in healthy Han Chinese subjects: the Cardiovascular Risk Survey. PLoS One 5: e13997.

Xie X, Ma YT, Yang YN, Li XM, et al. (2010b). Alcohol consumption and ankle-to-brachial index: results from the Cardiovascular Risk Survey. PLoS One 5: e15181.

Xie X, Ma YT, Yang YN, Fu ZY, et al. (2011). Polymorphisms in the SAA1 gene are associated with ankle-to-brachial index in Han Chinese healthy subjects. Blood Press 20: 232-238.

Yang W, Lu J, Weng J, Jia W, et al. (2010). Prevalence of diabetes among men and women in China. N. Engl. J. Med. 362: 1090-1101.

Yang YN, Xie X, Ma YT, Li XM, et al. (2012). Type 2 diabetes in Xinjiang Uygur autonomous region, China. PLoS One 7: e35270. 